\title{
Quantifying biofilm structure using image analysis
}

\author{
Xinmin Yang ${ }^{\mathrm{a}}$, Haluk Beyenal ${ }^{\mathrm{a}}$, Gary Harkin ${ }^{\mathrm{b}}$, Zbigniew Lewandowski $^{\mathrm{a}, \mathrm{c}, *}$ \\ ${ }^{a}$ Center for Biofilm Engineering, P.O. Box 173980, Room 336 EPS, Montana State University, Bozeman, MT 59717, USA \\ ${ }^{\mathrm{b}}$ Computer Science Department, Montana State University, Bozeman, MT 59717, USA \\ ${ }^{c}$ Department of Civil Engineering, Montana State University, Bozeman, MT 59717, USA
}

Received 5 April 1999; accepted 23 July 1999

\begin{abstract}
We have developed and implemented methods of extracting morphological features from images of biofilms in order to quantify the characteristics of the inherent heterogeneity. This is a first step towards quantifying the relationship between biofilm heterogeneity and the underlying processes, such as mass-transport dynamics, substrate concentrations, and species variations. We have examined two categories of features, areal, which quantify the relative magnitude of the heterogeneity and textural, which quantify the microscale structure of the heterogeneous elements. The feature set is not exhaustive and has been restricted to two-dimensional images to this point. Included in this paper are the methods used to extract the structural information and the algorithms used to quantify the data. The features discussed are porosity, fractal dimension, diffusional length, angular second moment, inverse difference moment and textural entropy. We have found that some features are better predictors of biofilm behavior than others and we discuss possible future directions for research in this area. (C) 2000 Elsevier Science B.V. All rights reserved.
\end{abstract}

Keywords: Biofilm; Image analysis; Structure; Heterogeneity quantification

\section{Introduction}

Many biofilm studies indicate that structural heterogeneity of biofilms may affect biofilm activity and intra-biofilm mass transfer dynamics (Keevil et al., 1993; Lewandowski et al., 1993; DeBeer et al., 1994; Walker et al., 1995; Yang and Lewandowski, 1995; White et al., 1996; Bishop, 1997; De Beer et al., 1997a; Suci et al., 1997; Wimpenny and Colasanti, 1997; Picioreanu et al., 1998a,b). To correlate biofilm structure to intra-biofilm mass transfer dynamics and biofilm activity it is necessary to quantify

\footnotetext{
*Corresponding author. Tel.: +1-406-994-5915; fax: +1-406994-6098.

E-mail address: zl@erc.montana.edu (Z. Lewandowski)
}

biofilm heterogeneity and to correlate it numerically with local mass transport rates and local respiration rates measured in natural biofilms. In this paper we present a set of techniques to quantify biofilm structure, with the goal of defining a set of parameters that would adequately represent biofilm morphology for analysis and modeling. The structural elements of the biofilm that are measured are called features and the quantified feature is referred to as a parameter.

Although biofilm structure has been studied extensively (Robinson et al., 1984; Brakenhoff et al., 1988; Christensen et al., 1989; Lawrence et al., 1991; Bremer et al., 1992; Caldwell et al., 1992; Dalton et al., 1994; Gjaltema et al., 1994; Tijhuis et al., 1994; Cao and Alaerts, 1995; Palmer and Caldwell, 1995; 
Stewart et al., 1995; van Loosdrecht et al., 1995; Swope and Flickinger, 1996; Gjaltema et al., 1997; Paulsen et al., 1997), the attempts to quantify the structure are much fewer and limited to calculating fractal dimension (Obert et al., 1990; Zahid and Ganczarczyk, 1994; Gibbs and Bishop, 1995; Hermanowics et al., 1995), and biofilm porosity (Zhang and Bishop, 1994; Holden et al., 1997). Our goal is to extend these works to a more comprehensive set of measures and to relate those measures to underlying processes that would uniquely describe biofilm structure. The working hypothesis is that in any

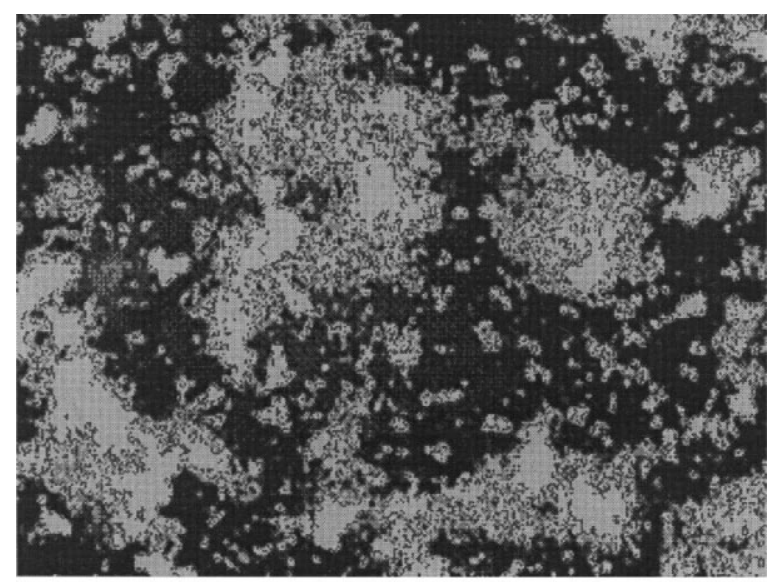

A

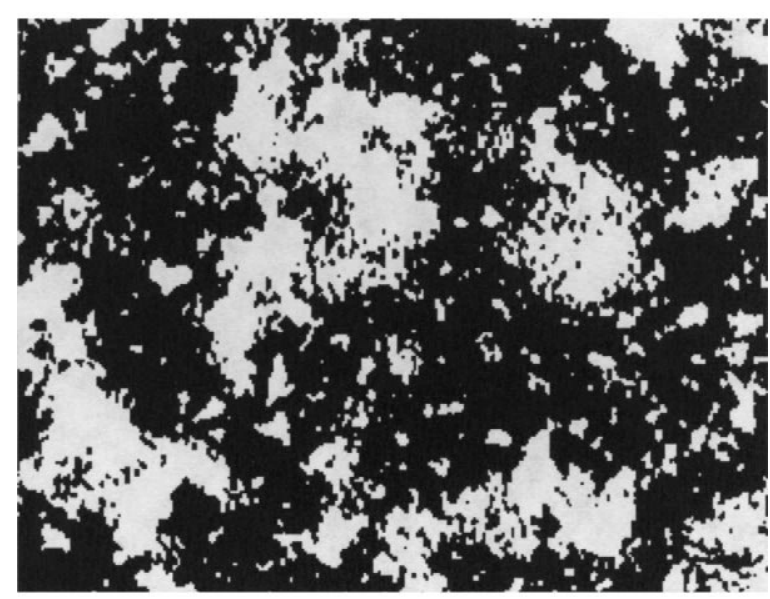

B

Fig. 1. Light microscope images of a biofilm. (A) Gray scale, (B) Binary. heterogeneous biofilm, there are a finite number of parameters that can be measured and used to quantify unique features of biofilm structure. This paper presents several parameters we were able to calculate from two-dimensional biofilm images taken by light microscopy or laser confocal microscopy. We developed algorithms to quantify biofilm structure from digitized computer images, and integrated these algorithms into a software package to automatically extract the features and produce the numerical parameters. The integration with underlying processes will be based on our current work where microelectrodes are used to quantify local intrabiofilm mass transport rates (Yang and Lewandowski, 1995; Beyenal et al., 1998) and local respiration rates (Lewandowski et al., 1992).

Cluster size and shape are examples of structural features. There are many features that one could choose to study, and an objective strategy is required to select those that are the most discerning between biofilms with different histories. The effectiveness of a feature in representing heterogeneity is based on its correlation with changes in the underlying processes that formed the biofilm and determined its behavior under known conditions. Features should be related in some manner to the underlying processes in order to be of scientific significance. It is reasonable, for example, to believe that the size of cell clusters or interstitial spaces might be associated with variations in population type and nutritional status, and that the shape of clusters might be associated with hydrodynamics.

\section{Quantifying structure parameters}

The parameters that describe biofilm structure can be classified as textural and areal. The textural parameters describe the microscale heterogeneity of the image and the areal parameters describe the morphological relationship between the size orientation and shape of surface features. The textural parameters studied are textural entropy, angular second moment and inverse difference moment, and the areal parameters are porosity, run length, fractal dimension and diffusion distance.

Fig. 1 shows a sample set of biofilm images. The 
textural parameters are calculated from a gray scale image (Fig. 1A) and the areal parameters are calculated from a binary image (Fig. 1B). The gray scale image is converted to a binary image by interactively selecting a threshold value that partitions the image into black and white pixels. The threshold value in this example is 150 while the pixel values range from 0 to 255 . If a pixel value in the image is less than 150 , it is set to one (black), otherwise it is set to zero (white). Each image is represented as a matrix and the elements of the matrix are the gray scale numerical value of the corresponding pixel. These values are used to calculate the measures of the structural parameters.

Thresholding is a subjective operation, where the operator attempts to find the value on the gray scale that best represents the distinction between biomass and void space. There are biomass components that may be too transparent to be detected, which introduces some error into the measurements. Also, there is inherent error in the shadows and image noise that cannot be directly compensated for. In a future project, we hope to quantify this error, as well as introduce mechanisms for reducing it.

Analysis of the images was accomplished with the Image Structure Analyzer (ISA), a software package developed by the Biofilm Structure-Function research group, Center for Biofilm Engineering, Montana State University for the purpose of quantifying biofilm structures. It was developed for the UNIX/ Motif environment in $\mathrm{C}++$ with all calculations done in double precision arithmetic. The calculation techniques presented in this paper were integrated into this computer program and the post-thresholding calculations are done automatically. In the near future the computer program may be available at the Center for Biofilm Engineering's Internet site www.erc.montana.edu.

\subsection{Textural parameters}

The textural parameters measure the microscale heterogeneity in the biofilm, by comparing the size, position and/or orientation of the biofilm constituents. The textural parameters are calculated from an 8-bit gray scale image, where each pixel has a value between 0 and 255 . Texture is broadly defined as the rate and direction of change of the chromatic properties of the image, and could be subjectively described as fine, coarse, smooth, random, rippled, irregular, etc. The calculations in the ISA were based on descriptions given by Haralick et al. (1973), with the textural parameters calculated from the normalized spatial dependence matrix.

If the image to be analyzed has dimensions $M$ rows by $N$ columns, and $N_{G}$ gray scale levels, then a triplet identifies each pixel:

$$
\begin{aligned}
(i, j, G), \quad 0 & \leq i \leq M-1, \quad 0 \leq j \leq N-1, \quad 0 \leq G \\
& \leq N_{G}-1
\end{aligned}
$$

The image I is a mapping from the spatial domain to the gray scale chromatic domain. The horizontal spatial dependence matrix is defined as:

$$
\begin{aligned}
& P_{H}=\left\{p_{H}(a, b)\right\}, \quad a, b \in\left[0, N_{G}-1\right] \\
& \begin{aligned}
p_{H}(a, b) & =\#\{((k, l), \quad(p, q)) \in(M, N), l=q,|k-p| \\
& =1, I(k, l)=a, I(p, q)=b\}
\end{aligned}
\end{aligned}
$$

$p_{H}(a, b)$ is the number of gray scale level changes in the image from grayscale level $a$ to grayscale level $b$ in pixels that are adjacent in a horizontal row either right-to-left or left-to-right.

Similarly, the vertical spatial dependence matrix is defined as:

$$
\begin{gathered}
P_{V}=\left\{p_{V}(a, b)\right\}, \quad a, b \in\left[0, N_{G}-1\right] \\
\begin{array}{c}
p_{V}(a, b)=\#\{((k, l), \quad(p, q)) \in(M \times N),|l-q| \\
=1, k=p, l(k, l)=a, l(p, q)=b\} \\
P_{H V}=P_{H}+P_{V}=\left\{p_{H}(a, b)+p_{V}(a, b)\right\}=\left\{p_{H V}(a, b)\right\}
\end{array}
\end{gathered}
$$

The normalized spatial dependence matrix is:

$$
P_{N}(a, b)=\left\{\frac{p_{H V}(a, b)}{\sum_{i, j} p_{H V}(a, b)}\right\}
$$

Each element $p(a, b)$ in the normalized spatial dependence matrix is the probability of a gray scale change from $a$ to $b$.

Example 1. Calculating the normalized spatial dependence matrix. A $4 \times 4$ biofilm image with four gray scale values, $0-3$, is shown below. 


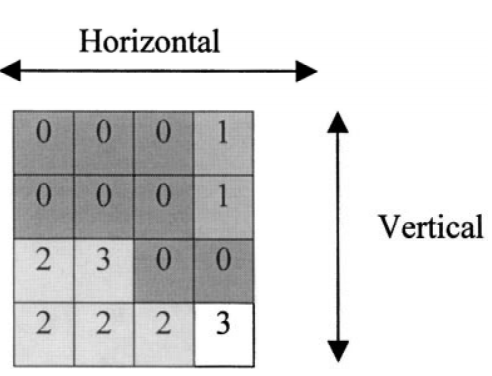

From the image, the horizontal spatial dependence matrix is:

$$
\begin{aligned}
P_{H} & =\left\{p_{H}(a, b)\right\} \\
& =\left[\begin{array}{llll}
p_{H}(0,0) & p_{H}(0,1) & p_{H}(0,2) & p_{H}(0,3) \\
p_{H}(1,0) & p_{H}(1,1) & p_{H}(1,2) & p_{H}(1,3) \\
p_{H}(2,0) & p_{H}(2,1) & p_{H}(2,2) & p_{H}(2,3) \\
p_{H}(3,0) & p_{H}(3,1) & p_{H}(3,2) & p_{H}(3,3)
\end{array}\right] \\
& =\left(\begin{array}{rrrr}
10 & 2 & 0 & 1 \\
2 & 0 & 0 & 0 \\
0 & 0 & 4 & 2 \\
1 & 0 & 2 & 0
\end{array}\right)
\end{aligned}
$$

$p_{H}(0,0)$ is the number of gray scale changes from 0 to 0 in horizontal direction. There are five 0 to 0 changes in the left-to-right direction and five in the right-to-left direction, so $p_{H}(0,0)=10 . p_{H}(0,1)$ is the number of gray scale changes from 0 to 1 in the horizontal direction. There are two such changes in the left-to-right direction and none in the right-to-left direction, so $p_{H}(0,1)=2$.

Similarly, the vertical spatial dependence matrix is:

$$
\begin{aligned}
P_{V} & =\left\{p_{V}(a, b)\right\} \\
& =\left[\begin{array}{llll}
p_{V}(0,0) & p_{V}(0,1) & p_{V}(0,2) & p_{V}(0,3) \\
p_{V}(1,0) & p_{V}(1,1) & p_{V}(1,2) & p_{V}(1,3) \\
p_{V}(2,0) & p_{V}(2,1) & p_{V}(2,2) & p_{V}(2,3) \\
p_{V}(3,0) & p_{V}(2,1) & p_{V}(2,2) & p_{V}(3,3)
\end{array}\right] \\
& =\left(\begin{array}{llll}
8 & 1 & 2 & 2 \\
1 & 2 & 0 & 0 \\
2 & 0 & 2 & 1 \\
2 & 0 & 1 & 0
\end{array}\right)
\end{aligned}
$$

The spatial dependence matrix is the sum of the horizontal and vertical spatial dependence matrix:

$$
P_{H V}=P_{H}+P_{V}=\left\{p_{H V}(a, b)\right\}=\left(\begin{array}{rrrr}
18 & 3 & 2 & 3 \\
3 & 2 & 0 & 0 \\
2 & 0 & 6 & 3 \\
3 & 0 & 3 & 0
\end{array}\right)
$$

Normalization is accomplished by summing the elements and dividing each element by the sum:

$$
\begin{aligned}
& \text { sum }=\sum_{a} \sum_{b} p_{H V}(a, b)=48 \\
& P_{N}=\{p(a, b)\}=\left(\begin{array}{llll}
0.375 & 0.063 & 0.042 & 0.063 \\
0.063 & 0.042 & 0 & 0 \\
0.042 & 0 & 0.125 & 0.063 \\
0.063 & 0 & 0.063 & 0
\end{array}\right)
\end{aligned}
$$

Based on the normalized spatial dependence matrix $P_{N}$, the textural parameters are defined as:

Textual entropy,

$\mathrm{TE}=-\sum_{a, b} \sum_{P(a, b) \neq 0} p(a, b) \ln (p(a, b))$

Angular second moment, $\quad \operatorname{ASM}=\sum_{a} \sum_{b}\left\{p(a, b)^{2}\right\}$

Inverse difference moment, IDM

$=\sum_{a} \sum_{b} \frac{1}{1+(a+b)^{2}} p(a, b)$

For this example, the parameter values are: $\mathrm{TE}=$ 2.072, $\mathrm{ASM}=0.185, \mathrm{IDM}=0.488$.

Each of these parameters measures the character of the cell clusters and interstitial space based on the likelihood that pixels of similar or dissimilar types would be neighbors. Textural entropy is a measure of the pure randomness in the gray scale image. The higher the textural entropy value, the more heterogeneous the biofilm. The angular second moment and inverse difference moment are similar measures but normalized for direction or distance respectively. Higher angular second moment values indicate more directional uniformity in the image, and inverse difference moment values indicate more or less variation in image contrast (Haralick et al., 1973).

\subsection{Areal parameters}

Areal parameters describe the morphological structures of biofilm. Each parameter measures a unique characteristic of either the cell cluster or 
interstitial space in the biofilm. These parameters are concerned with the size and shape of the constituent parts.

\subsubsection{Areal porosity}

The areal porosity is defined as the ratio of void area to total area and given by:

Areal porosity $=\frac{\text { Number of void pixels }}{\text { Total number of pixels }}$

Example 2. Calculation of areal porosity.

In the following binary image, the number of void (zero) pixels is 19 and total number of pixels is 36 , so the areal porosity is $19 / 36=0.528$.

\begin{tabular}{|l|l|l|l|l|l|}
\hline 0 & 0 & 0 & 0 & 0 & 0 \\
\hline 0 & 0 & 1 & 1 & 1 & 0 \\
\hline 0 & 1 & 1 & 1 & 1 & 1 \\
\hline 0 & 1 & 1 & 1 & 1 & 0 \\
\hline 0 & 1 & 1 & 1 & 1 & 0 \\
\hline 0 & 0 & 0 & 0 & 1 & 0 \\
\hline
\end{tabular}

\subsubsection{Run length}

The average horizontal run length is the average number of consecutive pixels with a value of one (cell cluster) in a row (horizontal). Similarly, the average vertical run length is the average number of consecutive pixels with value 1 in a column (vertical). The average run lengths measure the expected dimension of a cell cluster in each direction and is therefore a measure of the cluster size.

Example 3. Calculating average run length.

In the following binary biofilm image a one is a cluster pixel. The average horizontal run length is $(3+3+5+5) / 4=4$ (pixels) and the average vertical run length is $(2+4+4+4+2) / 5=3.2$ (pixels).

\begin{tabular}{|l|l|l|l|l|l|l|}
\hline 0 & 0 & 0 & 0 & 0 & 0 & 0 \\
\hline 0 & 0 & 1 & 1 & 1 & 0 & 0 \\
\hline 0 & 0 & 1 & 1 & 1 & 0 & 0 \\
\hline 0 & 1 & 1 & 1 & 1 & 1 & 0 \\
\hline 0 & 1 & 1 & 1 & 1 & 1 & 0 \\
\hline 0 & 0 & 0 & 0 & 0 & 0 & 0 \\
\hline 0 & 0 & 0 & 0 & 0 & 0 & 0 \\
\hline
\end{tabular}

\subsubsection{Diffusion distance}

The diffusion distance for a cluster is a measure of the distance from the cells in the cluster to interstitial space. Diffusion distance is related to both the sizes of the clusters and their general shape, as shown in Fig. 2.

Fig. 2 shows that for any cell in a cell cluster, the distance to each pixel that is on the boundary of the cluster can be calculated. Typically, the minimum distance is of interest as it is a measure of the distance to a source of nutrients for the cell. The diffusion distance is defined as the minimum distance from a cluster pixel to its nearest void pixel in an image. This study considers two different diffusion distance measures. The average diffusion distance is the average of the minimum distance from each cluster pixel to the nearest void pixel over all clusters pixels in the image. A larger diffusion distance indicates a higher distance that substrate has to diffuse in the cell cluster.

In ISA, an 'eight-point Euclidean distance mapping' algorithm is used to calculate average diffusion distance (Danielsson, 1980). For an $M \times N$ binary biofilm image, $(i, j)$ is an element of the image. $|P(i, j)|$ is the diffusion distance for the pixel at $(i, j)$ and is calculated as the Euclidian Distance of a cluster pixel from its nearest void pixel.

$$
\begin{aligned}
& P(i, j)=\left(P_{i}, P_{j}\right) \quad 0 \leq i \leq M-1, \quad 0 \leq j \leq N-1 \\
& |P(i, j)|=\sqrt{P_{i}^{2}+P_{j}^{2}}
\end{aligned}
$$

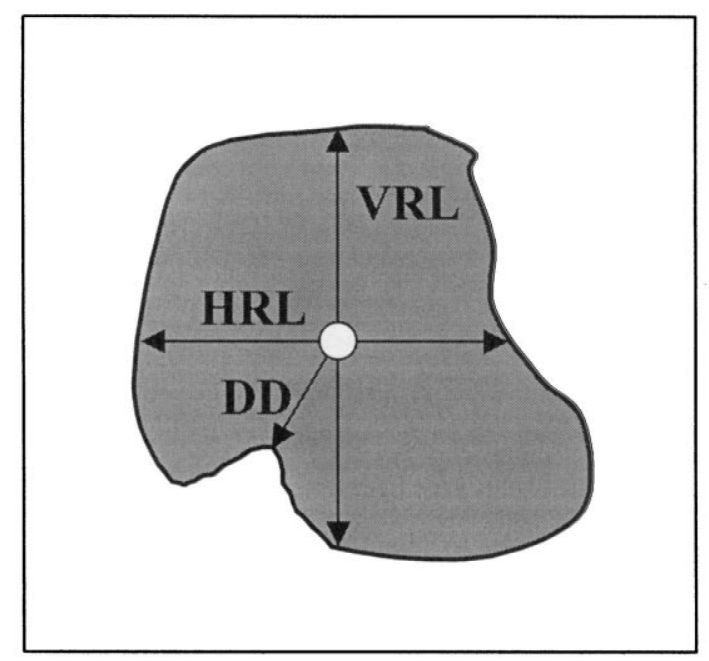

Fig. 2. Schematic diagram of a cell cluster (HRL: horizontal run length; VRL: vertical run length; DD: diffusion distance). 
The computer initializes an array of size $M \times N$ to a large value $(Z)$ to indicate infinite distance. Then it scans the array twice, once in the horizontal direction and once in the vertical direction. In each pass, it counts the number of pixels from a void pixel to a cluster pixel for each cluster pixel and maintains the minimum value found for the pixel. Upon termination, $|P(i, j)|$ based on the above definition gives the diffusion distance. Diffusion distances are only calculated for cluster pixels.

Example 4. Calculation of diffusion distance.

An example of the calculation of diffusion distance by means of Euclidean distance mapping is shown for a binary biofilm image.

\begin{tabular}{|l|l|l|l|l|l|}
\hline 0 & 0 & 0 & 0 & 0 & 0 \\
\hline 0 & 1 & 1 & 1 & 1 & 0 \\
\hline 0 & 1 & 1 & 1 & 1 & 0 \\
\hline 0 & 1 & 1 & 1 & 1 & 0 \\
\hline 0 & 0 & 0 & 0 & 0 & 0 \\
\hline
\end{tabular}

The initialized distance array is:

\begin{tabular}{|c|c|c|c|c|c|}
\hline 0,0 & 0,0 & 0,0 & 0,0 & 0,0 & 0,0 \\
\hline 0,0 & $\mathrm{Z}, \mathrm{Z}$ & $\mathrm{Z}, \mathrm{Z}$ & $\mathrm{Z}, \mathrm{Z}$ & $\mathrm{Z}, \mathrm{Z}$ & 0,0 \\
\hline 0,0 & $\mathrm{Z}, \mathrm{Z}$ & $\mathrm{Z}, \mathrm{Z}$ & $\mathrm{Z}, \mathrm{Z}$ & $\mathrm{Z}, \mathrm{Z}$ & 0,0 \\
\hline 0,0 & $\mathrm{Z}, \mathrm{Z}$ & $\mathrm{Z,Z}$ & $\mathrm{Z,Z}$ & $\mathrm{Z,Z}$ & 0,0 \\
\hline 0,0 & 0,0 & 0,0 & 0,0 & 0,0 & 0,0 \\
\hline
\end{tabular}

The first scan produces:

\begin{tabular}{|c|c|c|c|c|c|}
\hline 0,0 & 0,0 & 0,0 & 0,0 & 0,0 & 0,0 \\
\hline 0,0 & 1,0 & 2,0 & 2,0 & 1,0 & 0,0 \\
\hline 0,0 & 1,0 & 0,2 & 0,2 & 1,0 & 0,0 \\
\hline 0,0 & 0,1 & 0,1 & 0,1 & 0,1 & 0,0 \\
\hline 0,0 & 0,0 & 0,0 & 0,0 & 0,0 & 0,0 \\
\hline
\end{tabular}

The second scan produces:

\begin{tabular}{|c|c|c|c|c|c|}
\hline 0,0 & 0,0 & 0,0 & 0,0 & 0,0 & 0,0 \\
\hline 0,0 & 0,1 & 0,1 & 0,1 & 0,1 & 0,0 \\
\hline 0,0 & 1,0 & 0,2 & 0,2 & 1,0 & 0,0 \\
\hline 0,0 & 0,1 & 0,1 & 0,1 & 0,1 & 0,0 \\
\hline 0,0 & 0,0 & 0,0 & 0,0 & 0,0 & 0,0 \\
\hline
\end{tabular}

The final diffusion distance array is:

\begin{tabular}{|l|l|l|l|l|l|}
\hline 0 & 0 & 0 & 0 & 0 & 0 \\
\hline 0 & 1 & 1 & 1 & 1 & 0 \\
\hline 0 & 1 & 2 & 2 & 1 & 0 \\
\hline 0 & 1 & 1 & 1 & 1 & 0 \\
\hline 0 & 0 & 0 & 0 & 0 & 0 \\
\hline
\end{tabular}

The average diffusion distance $=(1+1+1+1+$ $1+2+2+1+1+1+1+1) / 12=1.17$ (pixels). The maximum diffusion distance is 2 , because the largest number in the above array is 2 .

\subsubsection{Fractal dimension}

Fractal geometry is a new area of mathematics that has been used by geologists, economists, and recently by microbiologists for quantifying the roughness of an object (Kaandorp, 1994; Russ, 1994). It is a mathematical system that allows objects to have a non-integral dimensionality, which is called the fractal dimension. In fractal geometry, the two-dimensional fractal dimension varies between 1 and 2 . The higher fractal dimension value, the more irregular the perimeter of the object. For the purposes of the analysis, the rougher the biofilm boundary, the higher the fractal dimension.

In ISA, a method called the Minkowski Sausage Method was used to calculate the fractal dimension (Russ, 1994), primarily because it relates naturally to image processing problems. The Minkowski Method uses dilation of the image to accomplish this.

The dilation process can be regarded as using a circle to continuously sweep through the perimeter, as shown in Fig. 3. Fig. 3(A) is the biofilm boundary and the dilation circle. The dilation circle is continuously swept through the boundary and thus completes the dilation process. Fig. 3(B) is the result of dilation using diameter 5. Fig. 3(C) is the result of 


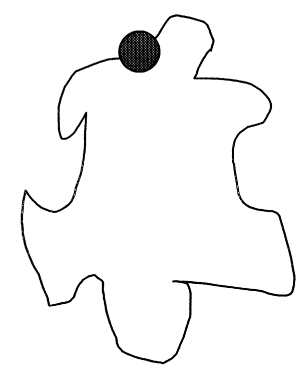

A

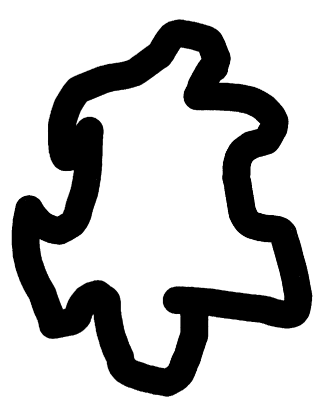

C

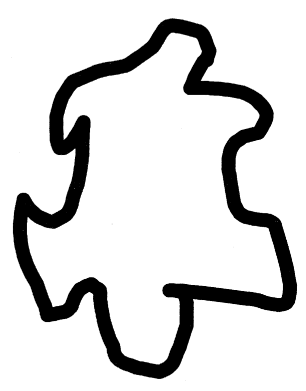

B

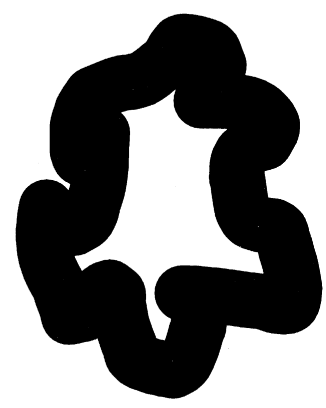

D
Fig. 3. Dilation using different diameter circles.

dilation using diameter 10. Fig. 3(D) is the result of dilation using diameter 20. The perimeter is measured by calculating the dilated area which is the number of black pixels in Fig. 3.

In ISA, the dilation process is completed using Euclidean distance mapping. Euclidean Distance Mapping, as used in the diffusion distance calculations, gives the distance of the cluster pixel to its nearest void pixel. If the boundary pixels are changed to zero and the other pixels one, and then applying the Euclidean Distance Mapping will calculate the distance to the boundary of that pixel. By choosing different radius values and counting the number of pixels that are smaller than this radius value the dilation area is acquired. Thus, perimeter $=$ dilated area/diameter.

Taking the logarithm and plotting the dilation circle diameter to the measured perimeter produces a straight line. The slope of the line can be easily calculated, and the fractal dimension is defined as (1-slope). It ranges from 1 to 2 . The higher the fractal dimension and rougher the biofilm boundary.
Example 5. Calculation of fractal dimension.

An example of calculating the fractal dimension is demonstrated for the binary image below.

\begin{tabular}{|l|l|l|l|l|l|l|l|}
\hline 0 & 0 & 0 & 0 & 0 & 0 & 0 & 0 \\
\hline 0 & 0 & 0 & 0 & 1 & 1 & 1 & 1 \\
\hline 0 & 0 & 0 & 0 & 1 & 1 & 1 & 1 \\
\hline 0 & 0 & 0 & 0 & 1 & 1 & 1 & 1 \\
\hline 0 & 0 & 0 & 1 & 1 & 1 & 1 & 1 \\
\hline 0 & 0 & 0 & 1 & 1 & 1 & 1 & 1 \\
\hline 0 & 0 & 0 & 1 & 1 & 1 & 1 & 1 \\
\hline 0 & 0 & 0 & 0 & 0 & 1 & 1 & 1 \\
\hline
\end{tabular}

First the boundary pixels are changed to zero and others to 1 (see below).

\begin{tabular}{|l|l|l|l|l|l|l|l|}
\hline 1 & 1 & 1 & 1 & 1 & 1 & 1 & 1 \\
\hline 1 & 1 & 1 & 1 & 0 & 0 & 0 & 0 \\
\hline 1 & 1 & 1 & 1 & 0 & 1 & 1 & 1 \\
\hline 1 & 1 & 1 & 1 & 0 & 1 & 1 & 1 \\
\hline 1 & 1 & 1 & 0 & 0 & 1 & 1 & 1 \\
\hline 1 & 1 & 1 & 0 & 1 & 1 & 1 & 1 \\
\hline 1 & 1 & 1 & 0 & 0 & 0 & 1 & 1 \\
\hline 1 & 1 & 1 & 1 & 1 & 0 & 1 & 1 \\
\hline
\end{tabular}

Then Euclidean Distance Mapping is used to produce an array in which each pixel has a value equal to its distance to the boundary.

\begin{tabular}{|c|c|c|c|c|c|c|c|}
\hline 4.1 & 3.2 & 2.2 & 1.4 & 1 & 1 & 1 & 1 \\
\hline 4 & 3 & 2 & 1 & 0 & 0 & 0 & 0 \\
\hline 3.6 & 3.0 & 2 & 1 & 0 & 1 & 1 & 1 \\
\hline 3.2 & 2.2 & 1.4 & 1 & 0 & 1 & 2 & 2 \\
\hline 3 & 2 & 1 & 0 & 0 & 1 & 2 & 2.8 \\
\hline 3 & 2 & 1 & 0 & 1 & 1 & 1.4 & 2.2 \\
\hline 3 & 2 & 1 & 0 & 0 & 0 & 1 & 2 \\
\hline 3.2 & 2.2 & 1.4 & 1 & 1 & 0 & 1 & 2 \\
\hline
\end{tabular}

The area is calculated from the result array by 
counting the total number of elements that are smaller than the radius value (radius value $=$ diameter/2).

For a radius value of 1.5 , the dilated area is 38 as shown below.

\begin{tabular}{|c|c|c|c|c|c|c|c|}
\hline 4.1 & 3.2 & 2.2 & 1.4 & 1 & 1 & 1 & 1 \\
\hline 4 & 3 & 2 & 1 & 0 & 0 & 0 & 0 \\
\hline 3.6 & 3.0 & 2 & 1 & 0 & 1 & 1 & 1 \\
\hline 3.2 & 2.2 & 1.4 & 1 & 0 & 1 & 2 & 2 \\
\hline 3 & 2 & 1 & 0 & 0 & 1 & 2 & 2.8 \\
\hline 3 & 2 & 1 & 0 & 1 & 1 & 1.4 & 2.2 \\
\hline 3 & 2 & 1 & 0 & 0 & 0 & 1 & 2 \\
\hline 3.2 & 2.2 & 1.4 & 1 & 1 & 0 & 1 & 2 \\
\hline
\end{tabular}

For a dilation radius value of 2.5 , the dilated area is 52 , as shown below.

\begin{tabular}{|c|c|c|c|c|c|c|c|}
\hline 4.1 & 3.2 & 2.2 & 1.4 & 1 & 1 & 1 & 1 \\
\hline 4 & 3 & 2 & 1 & 0 & 0 & 0 & 0 \\
\hline 3.6 & 3.0 & 2 & 1 & 0 & 1 & 1 & 1 \\
\hline 3.2 & 2.2 & 1.4 & 1 & 0 & 1 & 2 & 2 \\
\hline 3 & 2 & 1 & 0 & 0 & 1 & 2 & 2.8 \\
\hline 3 & 2 & 1 & 0 & 1 & 1 & 1.4 & 2.2 \\
\hline 3 & 2 & 1 & 0 & 0 & 0 & 1 & 2 \\
\hline 3.2 & 2.2 & 1.4 & 1 & 1 & 0 & 1 & 2 \\
\hline
\end{tabular}

For a dilation radius value of 3.5 , the dilated area is 61 , as shown below.

\begin{tabular}{|c|c|c|c|c|c|c|c|}
\hline 4.1 & 3.2 & 2.2 & 1.4 & 1 & 1 & 1 & 1 \\
\hline 4 & 3 & 2 & 1 & 0 & 0 & 0 & 0 \\
\hline 3.6 & 3.0 & 2 & 1 & 0 & 1 & 1 & 1 \\
\hline 3.2 & 2.2 & 1.4 & 1 & 0 & 1 & 2 & 2 \\
\hline 3 & 2 & 1 & 0 & 0 & 1 & 2 & 2.8 \\
\hline 3 & 2 & 1 & 0 & 1 & 1 & 1.4 & 2.2 \\
\hline 3 & 2 & 1 & 0 & 0 & 0 & 1 & 2 \\
\hline 3.2 & 2.2 & 1.4 & 1 & 1 & 0 & 1 & 2 \\
\hline
\end{tabular}

For a dilation radius value of 3.5 , the dilated area is 64 , as shown below.

\begin{tabular}{|c|c|c|c|c|c|c|c|}
\hline 4.1 & 3.2 & 2.2 & 1.4 & 1 & 1 & 1 & 1 \\
\hline 4 & 3 & 2 & 1 & 0 & 0 & 0 & 0 \\
\hline 3.6 & 3.0 & 2 & 1 & 0 & 1 & 1 & 1 \\
\hline 3.2 & 2.2 & 1.4 & 1 & 0 & 1 & 2 & 2 \\
\hline 3 & 2 & 1 & 0 & 0 & 1 & 2 & 2.8 \\
\hline 3 & 2 & 1 & 0 & 1 & 1 & 1.4 & 2.2 \\
\hline 3 & 2 & 1 & 0 & 0 & 0 & 1 & 2 \\
\hline 3.2 & 2.2 & 1.4 & 1 & 1 & 0 & 1 & 2 \\
\hline
\end{tabular}

Smaller dilation circle diameters give higher perimeters (area/diameter). The logarithmic plot of diameter versus perimeter gives a linear function as shown in Fig. 4. Fitting the data to a line gives a slope value of -0.5 , so the fractal dimension is $1-(-0.5)=1.5$.

\subsection{An application to real biofilm images}

To show the utility of ISA we analyzed two light microscopy images of a biofilm, 3 and 7 days old, (Fig. 5). The biofilm was viewed using UV light directed from the bottom using a Nikon ${ }^{\circledR}$ Diaphot 300 inverted microscope. Both images were acquired using the same illumination. Images were captured with a $\mathrm{COHU}^{\circledR}$ camera (Closed circuit, CA; model

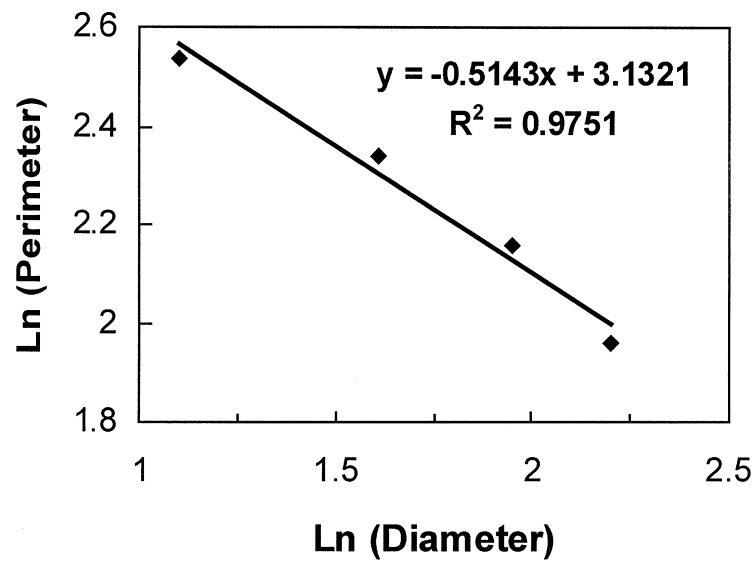

Fig. 4. $\operatorname{Ln}$ (diameter) versus $\operatorname{Ln}$ (perimeter). 


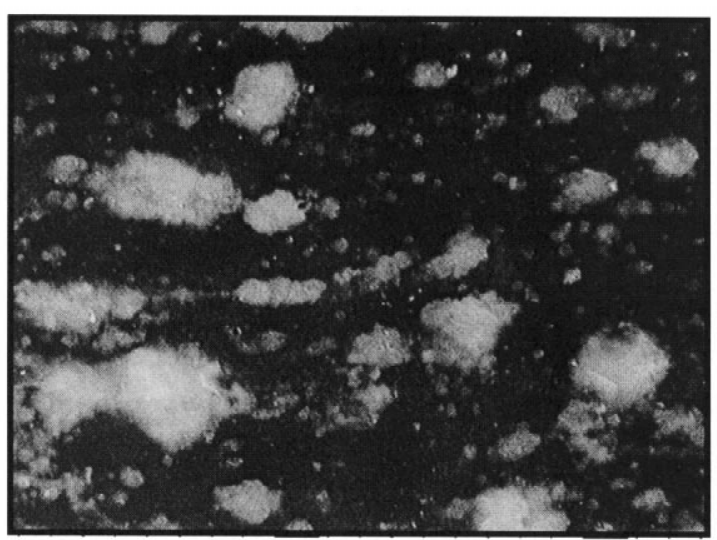

A

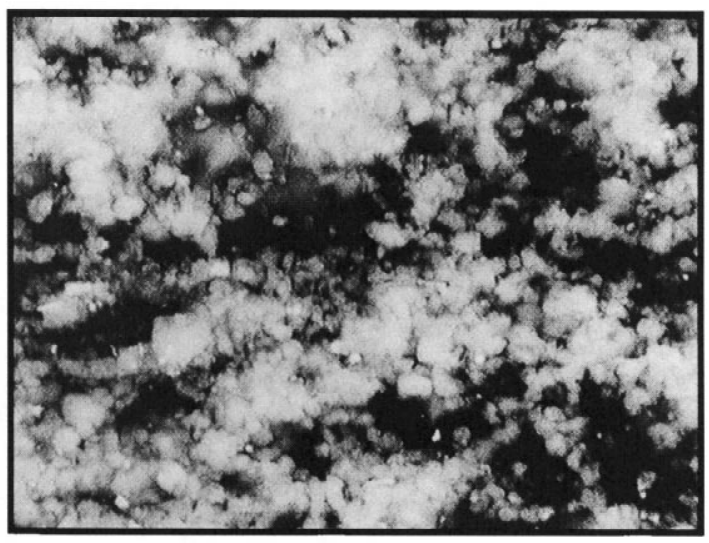

B

Fig. 5. (A) The third day biofilm; (B) the seventh day biofilm.

no: 2222-1040/0000) and Flashpoint ${ }^{\circledR}$ frame grabber (Integral technologies Inc., Indianapolis, Indiana) connected to a computer. The image size was $1568 \times$ $1176 \mu \mathrm{m}$ and the pixel size was $2.45 \mu \mathrm{m}$. The biofilm consisted of Pseudomonas aeruginosa, Pseudomonas fluorescens, and Klebsiella pneumoniae grown at $150 \mathrm{mg} / 1$ glucose concentration and 25 $\mathrm{cm} / \mathrm{s}$ flow velocity. Other information about growth conditions and experimental method for these biofilms are presented somewhere else (Xia et al., 1998). The images were thresholded manually; the white areas are cell clusters, the dark areas are
Table 1

Structure parameters calculated from the images of 3 and 7 days old biofilms

\begin{tabular}{lcc}
\hline Parameter & $\begin{array}{l}\text { 3 Days old } \\
\text { biofilm } \\
\text { (Fig. 5A) }\end{array}$ & $\begin{array}{l}\text { 7 Days old } \\
\text { biofilm } \\
\text { (Fig. 5B) }\end{array}$ \\
\hline Threshold value & 159 & 147 \\
Porosity & 0.66 & 0.17 \\
Textual entropy & 3.0014 & 3.3963 \\
Angular second moment & 0.0868 & 0.0475 \\
Inverse difference moment & 0.7079 & 0.6680 \\
Fractal dimension & 1.3720 & 1.3650 \\
Average horizontal run length & 10.59 & 37.94 \\
Average vertical run length & 9.56 & 35.01 \\
Average diffusion distance & 6.93 & 12.91 \\
Maximum diffusion distance & 44.05 & 58.24 \\
\hline
\end{tabular}

interstitial voids. The structure parameters calculated from the images are in Table 1.

The results in Table 1 show evaluation of structural parameters quantifying heterogeneity, from day 3 to 7. They can be analyzed from many points of view, depending on the particular question one wants to address. For example, the average diffusion distance reflects the length a substrate needs to travel to get to the middle of the microcolony. If the diffusion distance increases, chances are that the substrate will be exhausted before it reaches the middle section of the microcolony. It is then reasonable to expect that the middle of the microcolony will be occupied by different microorganisms than the outer boundary of the microcolony. Such a scenario can happen when nitrifiers convert ammonia to nitrate near the microcolony-bulk water interface and heterotrophs denitrify nitrate in the central part of the microcolony (De Beer et al., 1997b). Generally, the results in Table 1 reflect expectations. As time progresses biofilm porosity decreases and the cell clusters are bigger (have longer diffusion distances, horizontal and vertical run lengths). However, fractal dimension remained almost the same indicating that cluster surfaces remained constant. Textural entropy increased because of increased heterogeneity and the angular second moment and inverse difference moment decreased because cluster sizes and orientations were changed.

As a final test, we used the known shape and size 
of images as an independent check on the reliability of the structure data extracted by the ISA software (data not shown). The expected and calculated values of the structure parameters were the same.

\section{Conclusions}

The results of this ongoing study indicate that it is possible to extract and quantify morphological features of biofilms that can be related to underlying processes and conditions. In addition, other work has shown that it is possible to correlate the features to conditions such as steady-state behavior. Our goal is to improve the state-of-the-art in biofilm modeling and the understanding of the relationship between structure and function in biofilms.

The choice of two types of parameters, areal and textural, was based on a desire to apply methods from cluster theory (Jain, 1988) and texture modeling (Haralick and Shapiro, 1992). Although the images analyzed here are two-dimensional, the intent is to scale the parameterization to three dimensions. This complicates feature extraction but also makes it possible to correlate the results with other modes of data, such as hydrodynamics.

The immediate future of this work is in the investigation of other parametric measures and in determining analytical tests for the efficacy of those measures. In particular, it is necessary to develop techniques that will show that the selected features represent a majority of the variation in biofilm structure.

\section{Acknowledgements}

The research was supported by the cooperative agreement EED-8907039 between the National Science Foundation and Montana State University.

\section{References}

Beyenal, H., Tanyolaç, A., Lewandowski, Z., 1998. Measurement of local effective diffusivity in heterogeneous biofilms. Water Sci. Technol. 38, 171-178.
Bishop, P.L., 1997. Biofilm structure and kinetics. Water Sci. Technol. 36, 287-294.

Brakenhoff, G., van der Hoort, H., Baarslag, M., Oud, J., Zwart, R., var Driel, R., 1988. Visualization and analysis techniques for three-dimensional information acquired by confocal microscopy. Scan. Microsc. 2, 1831-1838.

Bremer, P., Geesey, G., Drake, B., 1992. Atomic force microscopy examination of the topography of a hydrated bacterial biofilm on a copper surface. Curr. Microbiol. 24, 223-230.

Caldwell, D., Korer, D., Lawrence, J., 1992. Confocal laser microscopy and digital image analysis in microbial ecology. Adv. Microbiol. Ecol. 12, 1-67.

Cao, Y.S., Alaerts, G.J., 1995. Influence of reactor type and shear stress on aerobic biofilm morphology, population and kinetics. Water Res. 29, 107-118.

Christensen, F.R., Kristensen, G.H., la Cour Jansen, J., 1989. Biofilm structure: An important and neglected parameter in wastewater treatment. Water Sci. Technol. 21, 805-814.

Dalton, H.M., Poulsen, L.K., Halasz, P., Angles, M.L., Goodman, A.E., Marshall, K.C., 1994. Substratum-induced morphological changes in a marine bacterium and their relevance to biofilm structure. J. Bacteriol. 11, 6090-6096.

Danielsson, P., 1980. Euclidean distance mapping. Comput. Graphics Imag. Proc. 14, 227-248.

De Beer, D., Stoodley, P., Roe, F., Lewandowski, Z., 1994. Effects of biofilm structures on oxygen distribution and mass transport. Biotechnol. Bioeng. 43, 1131-1138.

De Beer, D., Stoodley, P., Lewandowski, Z., 1997a. Measurement of local diffusion coefficients in biofilms by microinjection and confocal microscopy. Biotechnol. Bioeng. 53, 151-158.

De Beer, D., Schramm, A., Santegoeds, C.M., Kuhl, M., 1997b. A nitrite microsensor for profiling environmental biofilms. Appl. Environ. Microbiol. 63, 973-977.

Gibbs, J.T., Bishop, P.L., 1995. A method for describing biofilm surface roughness using geostatistical techniques. Water Sci. Technol. 32, 91-98.

Gjaltema, A., Arts, P.A.M., van Loosdrecht, M.C.M., Kuenen, J.G., Heijnen, J.J., 1994. Heterogeneity of biofilms in rotating annular reactors: Occurrence, structure, and consequences. Biotechnol. Bioeng. 44, 194-204.

Gjaltema, A., Vinke, L J., van Loosdrecht, M.C.M., Heijnen, J.J., 1997. Abrasion of suspended biofilm pellets in airlift reactors: Importance of shape, structure, and particle concentrations. Biotechnol. Bioeng. 53, 88-99.

Haralick, R.M., Shanmugam, K., Dinstein, I., 1973. Texture features for image classification. IEEE Trans. Syst. Man, Cybernet. SMC-3, 610-621.

Haralick, R., Shapiro, L. (Eds.), 1992. Computer and Robot Vision, Addison-Wesley, Reading, MA, pp. 453-493.

Hermanowics, S.W., Schindler, U., Wilderer, P., 1995. Fractal structure of biofilm: New tools for investigation of morphology. Water Sci. Technol. 32, 99-105.

Holden, P.A., Hunt, J.R., Firestone, M.K., 1997. Toluene diffusion and reaction in unstructured Pseudomonas putida biofilms. Biotechnol. Bioeng. 56, 656-670.

Jain, A., 1988. Algorithms For Clustering Data, Prentice Hall, New York. 
Kaandorp, J., 1994. Fractal Modeling Growth and Form in Biology, Springer, Berlin.

Keevil, C.W., Dowsett, A.B., Rogers, J., 1993. Legionella biofilms and their control. Society for Applied Bacteriology, 201-215, Technical series: microbial biofilms.

Lawrence, J.R., Korber, D.R., Hoyle, B.D., Costerton, J.W., Caldwell, D.E., 1991. Optical sectioning of microbial biofilms. J. Bacteriol. 10, 6558-6567.

Lewandowski, Z., Altobelli, S.A., Majors, P.D., Fukushima, E., 1992. NMR imaging of hydrodynamics near microbially colonized surfaces. Water Sci. Technol. 26, 577-584.

Lewandowski, Z., Stoodley, P., Altobelli, S., Fukushima, E., 1993. Hydrodynamics and kinetics in biofilm systems: recent advances and new problems. In: Proc. of the 2nd IAWQ International Specialized Conference on Biofilm Reactors, September 29-October 1, 1993, Paris, France, pp. 313-319.

Obert, M., Pfeifer, P., Sernetz, M., 1990. Microbial growth patterns described by fractal geometry. J. Bacteriol. 1, 11801185.

Palmer, R.J., Caldwell, D.E., 1995. A flowcell for study of plaque removal and regrowth. J. Microbiol. Methods 24, 171-182.

Paulsen, J.E., Oppen, E., Bakke, R., 1997. Biofilm morphology in porous media. A study with microscopic and image techniques. Water Sci. Technol. 36, 1-9.

Picioreanu, C., van Loosdrecht, M.C.M., Heijnen, J.J., 1998a. A new combined differential-discrete cellular automaton approach for biofilm modeling: Application for growth in gel beads. Biotechnol. Bioeng. 57, 718-731.

Picioreanu, C., van Loosdrecht, M.C.M., Heijnen, J.J., 1998 b. Mathematical modeling of biofilms structure with a hybrid differential-discrete cellular automaton approach. Biotechnol. Bioeng. 58, 101-116.

Robinson, R.W., Akin, D.E., Nordstedt, R.A., Thomas, M.V., Aldrich, H.C., 1984. Light and electron microscopic examinations of methane producing biofilms from anaerobic fixed bed reactors. Appl. Environ. Microbiol. 48, 127-136.

Russ, J., 1994. Fractal Surfaces, Plenum Press, New York.

Stewart, P.S., Murga, R., Srinivasan, R., de Beer, D., 1995. Biofilm structural heterogeneity visualized by three microscopic methods. Water Res. 29, 2006-2009.
Suci, P.A., Siedlecki, K.J., Palmer, R.J., White, D.C., Geesey, G.G., 1997. Combined light microscopy and attenuated total reflection Fourier transform infrared spectroscopy for integration of biofilm structure, distribution, and chemistry at solidliquid interfaces. Appl. Environ. Microbiol. 63, 4600-4603.

Swope, K.L., Flickinger, M.C., 1996. The use of confocal scanning laser microscopy and other tools to characterize Escherichia coli in a high cell density synthetic biofilm. Biotechnol. Bioeng. 52, 340-356.

Tijhuis, L., Rekswinkel, E., van Loosdrecht, M.C.M., Heijnen, J.J., 1994. Dynamics of population and biofilm structure in the biofilm airlift suspension reactor for carbon and nitrogen removal. Water Sci. Technol. 29, 377-384.

van Loosdrecht, M.C.M., Eikelboom, D., Gjaltema, A., Mulder, A., Tijhuis, L., Heijnen, J.J., 1995. Biofilm structures. Water Sci. Technol 32, 35-43.

Walker, J.T., Mackerness, C.W., Rogers, J., Keevil, C.W., 1995. Heterogeneous mosaic: a haven for waterborne pathogens. In: Lappin-Scott, H.M., Costerton), J.W. (Eds.), Microbial Biofilms, Cambridge University Press, Cambridge, UK, pp. 196-204.

White, D.C., Arrage, A.A., Nivens, D.E., Palmer, R.J., Rice, J.F., Sayler, G.S., 1996. Biofilm ecology: on-line methods bring new insights into MIC and microbial biofouling. Biofouling 10, $1-3$.

Wimpenny, J.W., Colasanti, R., 1997. A unifying hypothesis for the structure of microbial biofilms based on cellular automaton models. FEMS Microbiol. Ecol. 22, 1-16.

Xia, F., Beyenal, H., Lewandowski, Z., 1998. An electrochemical technique to measure local flow velocity in biofilms. Water Res. 32, 3631-3636.

Yang, S., Lewandowski, Z., 1995. Measurement of local mass transfer coefficient in biofilms. Biotechnol. Bioeng. 48, 737744.

Zahid, W.M., Ganczarczyk, J.J., 1994. Fractal properties of the RBC biofilm structure. Water Sci. Technol. 29, 271-279.

Zhang, T.C., Bishop, P.L., 1994. Density, porosity, and pore structure of biofilms. Water Res. 28, 2267-2277. 